\title{
Editorial
}

\section{Jahresbericht der Herausgeber 1996/1997}

Seit 1996 treffen sich Herausgeber und Beiräte der Zeitschrift für Orthopädie alljährlich anläßlich des Deutschen Orthopäden Kongresses. Dabei werden Möglichkeiten und Maßnahmen besprochen, wie das Konzept der Zeitschrift verbessert und den Markterfordernissen angepaßt werden kann. Als wesentliche Ziele der Zeitschrift wurden von den Herausgebern die wissenschaftliche Qualität und die Attraktivität für den Leser herausgestellt (Niethard, 1996).

Die wissenschaftliche Qualität wird durch den ImpactFactor gemessen. Demnach ist die Zeitschrift für Orthopädie nach der letzten Erhebung aus dem Jahre 1995 das beste nicht englischsprachige Journal und steht an 20 . Stelle des Journal Rankings für das Fach Orthopädie (Tab. 1). Der Impact-Faktor ist in seiner Bedeutung nach wie vor umstritten (Niethard, 1997). Die Deutsche Gesellschaft für Unfallchirurgie hat in ihrem Beschluß vom 25. 6. 1997 zum Ausdruck gebracht, daß der Impact-Faktor nicht als $\mathrm{Maß}$ wissenschaftlicher Leistungen geeignet ist. Herausgeber und Beiräte der Zeitschrift für Orthopädie sind ebenso der Meinung, daß der Impact-Faktor für die Bewertung von Kandidaten im Rahmen von Habilitationsverfahren nicht als ausschließliches Kriterium herangezogen werden kann. Dessen ungeachtet ist der Impact-Faktor die einzige Maßzahl für die qualitative Bewertung wissenschaftlicher Journale und aus diesem Grund derzeit unverzichtbar. Es wird weiterhin das Ziel der Zeitschrift für Orthopädie sein, den Rang in der Liste der besten 20 orthopädischen Publikationsorgane zu festigen.

Die wissenschaftliche Qualität einer Zeitschrift ergibt sich auch aus der Bearbeitungsdauer der eingereichten Manuskripte. Die Anzahl der eingegangenen Manuskripte ist im Jahr 1996/1997 um 20\% gegenüber den Vorjahren gestiegen. Die durschschnittliche Bearbeitungsdauer der Manuskripte beträgt derzeit 4 Wochen und liegt damit im angestrebten Rahmen. Die Schriftleitung dankt allen Gutachtern für den zeitlichen Einsatz und die sorgfältige Aufarbeitung der Manuskripte. In Einzelfällen ist eine Verkürzung der Begutachtung möglich.

Die Qualität der Manuskripte wird nach formalen und inhaltlichen Gesichtspunkten bewertet. Die formalen Anforderungen sind in den Richtlinien für die Autoren formuliert und werden in zunehmendem Umfang eingehalten. Aus diesem Grund müssen nur noch wenige Manuskripte aus formalen Gründen abgewiesen werden. Dessen ungeachtet beträgt die Rate primär angenommener Manuskripte derzeit nur 5\%. 35\% der Manuskripte werden vorwiegend aus inhaltlichen Gründen primär abgelehnt. $60 \%$ der eingereichten Arbeiten werden zur Re- vision an die Autoren zurückgeschickt. Im Revisionsverfahren ist eine neuerliche Begutachtung des Manuskriptes erforderlich. Zeitliche Verzögerungen gehen dabei meistens zu Lasten der Autoren.

Ein besonderes Augenmerk wird von Herausgebern und Beiräten der wissenschaftlichen Lauterkeit geschenkt. Die zunehmende Anzahl von gefälschten und Doppelpublikationen veranlaßt die Herausgeber der Zeitschrift für Orthopädie dazu, sich den Richtlinien in englischsprachigen Journalen anzuschließen. In einem Editorial des Jornal of Bone and Joint Surgery wird über einen besonders bedauerlichen Fall von Falschpublikation berichtet, der zur Zurückziehung eines Manuskriptes im Journal geführt hat (Fulford, 1997). Im Rahmen eines Austausches von regelmäßigen Journalen wurden Doppelpublikationen für die Zeitschrift für Orthopädie und die Acta Orthopaedica Scandinavica entdeckt. Die versuchte Doppelveröffentlichung wurde von der Schriftleitung der Acta Orthop. Scand. als schwerwiegendes Fehlverhalten interpretiert. Um derartigen Entwicklungen vorzubeugen, werden die Herausgeber auch für die Zeitschrift für Orthopädie eine Erklärung der Autoren anfordern, daß das Manuskript noch nicht an anderer Stelle veröffentlicht wurde. Dies gilt nicht für Manuskripte, die auf Anforderung der Herausgeber erstellt werden. Diese Anforderungen werden in die Richtlinien für die Autoren aufgenommen und sind damit unverzichtbarer Bestandteil der formalen Vorbedingungen für die Annahme des Manuskriptes.

Wissenschaftliche Qualität und Attraktivität der Zeitschrift sind durch die Publikation der Kongreßabstracts verbessert worden. Die Zusammenfassungen lagen bereits mit Beginn des Kongresses gedruckt vor, wofür dem Kongreßpräsidenten besonders zu danken ist. Damit ist die Zeitschrift für Orthopädie außerordentlich aktuell, ein Faktor, der der deutschsprachigen Orthopädie besonders zugute kommt, da die Abstracts erstmals seit längerer Zeit wieder zitierfähig sind.

Die Attraktivität der Zeitschrift für Orthopädie konnte im Berichtsjahr 1996/1997 auch durch die Einbindung der „Orthopädie aktuell“ gesteigert werden. In diesem Zusammenhang gebührt Herrn Prof. Krämer und Frau Dr. Rita Engelhardt als freie journalistische Mitarbeiter besonderer Dank für die Ausgestaltung dieses Teiles. Die steigende Abonnentenzahl ist Beleg dafür, daß das Konzept wohlwollend aufgegriffen wurde. Herausgebern und Beiräten ist es gelungen, entsprechend ihrer Zielvorstellung die Zeitschrift für Orthopädie wissenschaftlich effizient, lehrreich und für möglichst viele Orthopäden interessant zu machen. 


\section{Literatur}

Fulford, Ph.: Retraction of a paper - Editorial. J. Bone Joint Surg. 79-B (1997) 705-706

Niethard, F. U., M. H. Hackenbroch, R. Kotz, J. Krämer, W. Puhl: Editorial. Z. Orthop. 125 (1996) 1-2

Niethard, F. U.: Im Pakt mit dem Impact-Faktor? - Editorial. Z. Orthop. 134 (1996) 293-294

Niethard, F. U.: „Virtuelle Realität in der publizierten Wissenschaft?“-Editorial. Z. Orthop. 134 (1996) 481-482

Weller, S., M. Wachinger: Impact-Faktor - oder careful human judgement? - Editorial. Act. Traumatol. 27 (1997) 123-124

\section{F. U. Niethard, Aachen}

Tab. 1 Journal Citation Reports (JCR) on CD-ROM - 1995 Science Edition Journal Rankings Sorted by Impact Factor (Filtered by ORTHOPEDICS)

\begin{tabular}{|c|c|c|c|c|c|c|c|}
\hline Rank & $\begin{array}{l}\text { Journal } \\
\text { Abbreviation }\end{array}$ & ISSN & $\begin{array}{l}1995 \\
\text { Total Cites }\end{array}$ & $\begin{array}{l}\text { Impact } \\
\text { Factor }\end{array}$ & $\begin{array}{l}\text { Immed. } \\
\text { Index }\end{array}$ & $\begin{array}{l}1995 \\
\text { Articles }\end{array}$ & $\begin{array}{l}\text { Cited } \\
\text { Half-Life }\end{array}$ \\
\hline 1 & OSTEOPOROSIS INT & $0937-941 X$ & 1065 & 2.906 & 0.250 & 60 & 2.8 \\
\hline 2 & J BONE JOINT SURG AM & $0021-9355$ & 9565 & 1.602 & 0.141 & 227 & $>10.0$ \\
\hline 3 & J BONE JOINT SURG BR & $0301-620 x$ & 4735 & 1.160 & 0.198 & 202 & $>10.0$ \\
\hline 4 & J ORTHOPAED RES & $0736-0266$ & 1707 & 1.050 & 0.172 & 116 & 5.8 \\
\hline 5 & PHYS THER & $0031-9023$ & 1210 & 0.798 & 0.176 & 108 & 7.3 \\
\hline 6 & ACTA ORTHOP SCAND & $0001-6470$ & 2419 & 0.744 & 0.099 & 202 & $>10.0$ \\
\hline 7 & CLIN ORTHOP RELAT R & $0009-921 x$ & 9353 & 0.687 & 0.063 & 414 & 9.1 \\
\hline 8 & SPINE & $0362-2436$ & 3732 & 0.588 & 0.154 & 422 & 6.6 \\
\hline 9 & ARTHROSCOPY & 0749-8063 & 509 & 0.524 & 0.016 & 122 & 5.2 \\
\hline 10 & J PEDIATR ORTHOPED & $0271-6798$ & 742 & 0.473 & 0.051 & 138 & 6.8 \\
\hline 11 & ORTHOP CLIN N AM & 0030-5898 & 907 & 0.459 & 0.000 & 62 & 8.7 \\
\hline 12 & PARAPLEGIA & $0031-1758$ & 608 & 0.398 & 0.099 & 141 & 6.1 \\
\hline 13 & CLIN BIOMECH & $0268-0033$ & 148 & 0.355 & 0.000 & 66 & 4.8 \\
\hline 14 & J LONG-TERM EFF MED & $1050-6934$ & 30 & 0.325 & 0.000 & 6 & \\
\hline 15 & J HAND SURG-AM & $0363-5023$ & 1465 & 0.322 & 0.026 & 193 & 8.6 \\
\hline 16 & FOOT ANKLE INT & $1071-1007$ & 335 & 0.268 & 0.000 & 145 & 6.2 \\
\hline 17 & J SPINAL DISORD & 0895-0385 & 174 & 0.253 & 0.000 & 82 & 3.9 \\
\hline 18 & PROSTHET ORTHOT INT & 0309-3646 & 71 & 0.200 & 0.050 & 20 & \\
\hline 19 & HAND CLIN & 0749-0712 & 159 & 0.183 & 0.000 & 55 & 5.7 \\
\hline 20 & ORTHOPEDICS & $0147-7447$ & 521 & 0.162 & 0.013 & 155 & 6.5 \\
\hline 20 & Z ORTHOP GRENZGEB & $0044-3220$ & 253 & 0.162 & 0.010 & 98 & 8.9 \\
\hline 22 & ARCH ORTHOP TRAUM SU & 0936-8051 & 393 & 0.160 & 0.016 & 62 & 8.6 \\
\hline 23 & J HAND SURG-BRIT EUR & $0266-7681$ & 280 & 0.158 & 0.018 & 170 & 6.1 \\
\hline 24 & J ORTHOP SPORT PHYS & 0190-6011 & 167 & 0.151 & 0.000 & 74 & 6.6 \\
\hline 25 & ORTHOPADE & $0085-4530$ & 111 & 0.150 & 0.000 & 70 & 6.1 \\
\hline 26 & J PEDIATR ORTHOP B & $1060-152 X$ & 15 & 0.141 & 0.000 & 40 & \\
\hline 27 & INT ORTHOP & 0341-2695 & 301 & 0.139 & 0.000 & 88 & 5.9 \\
\hline 28 & J MANIP PHYSIOL THER & $0161-4754$ & 97 & 0.126 & 0.000 & 78 & \\
\hline 29 & REV CHIR ORTHOP & $0035-1040$ & 189 & 0.088 & 0.013 & 77 & $>10.0$ \\
\hline 30 & J ORTHOP RHEUMATOL & $0951-9580$ & 21 & 0.063 & 0.000 & 34 & \\
\hline 31 & J AM PODIAT MED ASSN & & 117 & 0.053 & 0.000 & 103 & 6.6 \\
\hline
\end{tabular}

